\title{
A Note on $(h, q)$-Genocchi Polynomials and Numbers of Higher Order
}

\author{
Lee-Chae Jang, ${ }^{1}$ Kyung-Won Hwang, ${ }^{2}$ and Young-Hee $\mathrm{Kim}^{3}$ \\ ${ }^{1}$ Department of Mathematics and Computer Science, KonKuk University, Chungju 138-701, South Korea \\ 2 Department of Mathematics, Dong-A University, Busan 604-714, South Korea \\ ${ }^{3}$ Division of General Education, Kookmin University, Seoul 136-702, South Korea
}

Correspondence should be addressed to Lee-Chae Jang, leechae.jang@kku.ac.kr

Received 10 July 2009; Revised 5 December 2009; Accepted 11 February 2010

Academic Editor: Patricia J. Y. Wong

Copyright (C) 2010 Lee-Chae Jang et al. This is an open access article distributed under the Creative Commons Attribution License, which permits unrestricted use, distribution, and reproduction in any medium, provided the original work is properly cited.

We investigate several arithmetic properties of $(h, q)$-Genocchi polynomials and numbers of higher order.

\section{Introduction and Preliminaries}

Recently, Kim [1] studied $q$-Genocchi and Euler numbers using Fermionic $q$-integral and introduced related applications. Kim [2] also gives the $q$-extensions of the Euler numbers which can be viewed as interpolating of $q$-analogue of Euler zeta function at negative integers and gives Bernoulli numbers at negative integers by interpolating Riemann zeta function. These numbers are very useful for number theory and mathematical physics. Kim $[3,4]$ studied $q$-Bernoulli numbers and polynomials related to Gaussian binomial coefficient and studied also some identities of $q$-Euler polynomials and $q$-stirling numbers. Kim [5] made Dedekind DC sum in the meaning of extension of Dedekind sum or Hardy sum and introduced lots of interesting results. The purpose of this paper is to investigate several arithmetic properties of $(h, q)$-Genocchi polynomials and numbers of higher order.

Let $p$ be a fixed odd prime. Throughout this paper $\mathbb{Z}, \mathbb{Z}_{p}, \mathbb{Q}_{p}$, and $\mathbb{C}_{p}$ will, respectively, denote the ring of rational integers, the ring of $p$-adic rational integers, the field of $p$-adic rational numbers, and the completion of algebraic closure of $\mathbb{Q}_{p}$. Let $v_{p}$ be the normalized exponential valuation of $\mathbb{C}_{p}$ with $|p|_{p}=p^{-v_{p}(p)}=p^{-1}$. When one talks of $q$-extension, $q$ is variously considered as an indeterminate, a complex number $q \in C$, or a $p$-adic number $q \in \mathbb{C}_{p}$. If $q \in \mathbb{C}$, one normally assumes $|q|<1$. If $q \in \mathbb{C}_{p}$, then we assume $|q-1|_{p}<p^{-1 /(p-1)}$, 
so that $q^{x}=\exp (x \log q)$ for $|x|_{p} \leq 1$. We also use the notations

$$
[x]_{q}=\frac{1-q^{x}}{1-q}, \quad[x]_{-q}=\frac{1-(-q)^{x}}{1+q}
$$

for all $x \in \mathbb{Z}_{p}$ (see $\left.[5-12]\right)$. Hence, $\lim _{q \rightarrow 1}[x]_{q}=x$.

Let $d$ be a fixed positive integer with $(p, d)=1$. We now set

$$
X=\lim _{\overleftarrow{N}} \frac{\mathbb{Z}}{d p^{N} \mathbb{Z}^{\prime}}, \quad X^{*}=\bigcup_{\substack{0<a<d p \\(a, p)=1}} a+d p \mathbb{Z}_{p}, \quad a+d p^{N} \mathbb{Z}_{p}=\left\{x \in X \mid x \equiv a\left(\bmod p^{N}\right)\right\},
$$

where $a \in \mathbb{Z}$ lies in $0 \leq a<d p^{N}$. For any $N \in \mathbb{N}$, we set

$$
\mu_{q}\left(a+d p^{N} \mathbb{Z}_{p}\right)=\frac{(-q)^{a}}{\left[d p^{N}\right]_{-q}}
$$

and this can be extended to a distribution on $\mathbb{Z}_{p}$.

We say that $f$ is a uniformly differentiable function at a point $a \in \mathbb{Z}_{p}$ and write $f \in$ $U D\left(\mathbb{Z}_{p}\right)$, if the difference quotients $F_{f}(x, y)=((f(x)-f(y)) /(x-y))$ have a limit $f^{\prime}(a)$ as $(x, y) \rightarrow(a, a)$ (cf. [13-23]).

For $f \in U D\left(\mathbb{Z}_{p}\right)$, the $p$-adic invariant integral on $\mathbb{Z}_{p}$ is defined as

$$
I(f)=\int_{X} f(x) d \mu(x)=\lim _{N \rightarrow \infty} \sum_{x=0}^{d p^{N}-1} f(x)(-1)^{x}
$$

(see [14, 23]). Let $n \in N$ and $f_{n}(x)=f(x+n)$. From (1.4), we have

$$
I\left(f_{n}\right)+I(f)=2 \sum_{l=0}^{n-1}(-1)^{l} f(l)
$$

The $p$-adic integral has been used in many areas such as mathematics, physics, probability theory, dynamical systems, and biological models. Especially, Khrennikov [24-26] applied to many areas using ingenious technique. The Genocchi numbers $G_{n}$ and polynomials $G_{n}(x)$ are defined by the generating functions as follows:

$$
\frac{2 t}{e^{t}+1}=\sum_{n=0}^{\infty} G_{n} \frac{t^{n}}{n !}, \quad \frac{2 t}{e^{t}+1} e^{x t}=\sum_{n=0}^{\infty} G_{n}(x) \frac{t^{n}}{n !}
$$

(see $[5,7,15])$. The $q$-extension of Genocchi numbers are defined by

$$
F_{q}(t)=t \sum_{m=0}^{\infty}(-1)^{m} q^{m} e^{[m]_{q} t}=\sum_{n=0}^{\infty} G_{n, q} \frac{t^{n}}{n !}
$$


(see $[1,2])$, and the $q$-extension of Genocchi polynomials is also given by

$$
G_{n, q}(x)=\sum_{l=0}^{n}\left(\begin{array}{l}
n \\
l
\end{array}\right) q^{l x} G_{l, q}[x]_{q}^{n-l}
$$

In Section 2, we investigate several arithmetic properties of $(h, q)$-Genocchi polynomials and numbers of higher order.

\section{2. $(h, q)$-Genocchi Numbers of Higher Order}

Let $h, k \in N$ and $q \in C$ with $|q-1|_{p}<p^{-1 /(p-1)}$. The $(h, q)$-Genocchi polynomials $G_{m, q}^{(h, k)}(x)$ of order $k$ are defined as

$$
t^{k} \int_{\mathbb{Z}_{p}} \cdots \int_{\mathbb{Z}_{p}} e^{\left[x_{1}+\cdots x_{k}+x\right]_{q} t} q^{(h-1) x_{1}+\cdots+(h-k) x_{k}} d \mu_{q}\left(x_{1}\right) \cdots d \mu_{q}\left(x_{k}\right)=\sum_{m=0}^{\infty} G_{m, q}^{(h, k)}(x) \frac{t^{m}}{m !}
$$

where $\int_{\mathbb{Z}_{p}} f(x) d \mu_{q}(x)=\lim _{N \rightarrow \infty}\left(1 /\left[p^{N}\right]_{-q}\right) \sum_{x=0}^{p^{N}-1} f(x)(-q)^{x}$. It is easily to see that $G_{0, q}^{(h, k)}(x)=$ $\cdots=G_{k-1, q}^{(h, k)}(x)=0$ for each $h \in \mathbb{Z}$ and $k \in \mathbb{N}$. From (2.1), we can obtain the following theorem.

Theorem 2.1. Let $h \in \mathbb{Z}$ and $m, k \in \mathbb{N}$. Then for all $x \in \mathbb{Z}_{p}$,

$$
\frac{k !}{(m+k) !} G_{m+k, q}^{(h, k)}(x)=\int_{\mathbb{Z}_{p}} \cdots \int_{\mathbb{Z}_{p}}\left[x_{1}+\cdots x_{k}+x\right]_{q}^{m} q^{(h-1) x_{1}+\cdots+(h-k) x_{k}} d \mu_{q}\left(x_{1}\right) \cdots d \mu_{q}\left(x_{k}\right) .
$$

From Theorem 2.1, if we take $k=-m(m>0)$, then

$$
\frac{1}{m !\left(\begin{array}{c}
m+k \\
m
\end{array}\right)} G_{m+k, q}^{(-m, k)}(x)=\int_{\mathbb{Z}_{p}} \cdots \int_{\mathbb{Z}_{p}}\left[x_{1}+\cdots x_{k}+x\right]_{q}^{m} q^{-(m+1) x_{1}-\cdots-(m+k) x_{k}} d \mu_{q}\left(x_{1}\right) \cdots d \mu_{q}\left(x_{k}\right) .
$$

Now, we define $(h, q)$-Genocchi number of higher order as follows:

$$
G_{m, q}^{(-m, k)}=G_{m, q}^{(-m, k)}(0)
$$

From (2.4), we can derive the following theorem. 
Theorem 2.2. Let $h \in \mathbb{Z}$ and $m, k \in \mathbb{N}$. Then one has

$$
\begin{aligned}
\frac{G_{m+k, q}^{(-m, k)}}{m !\left(\begin{array}{c}
m+k \\
m
\end{array}\right)} & =\lim _{N \rightarrow \infty} \frac{1}{\left[p^{N}\right]_{-q}^{k}} \sum_{x_{1}=0}^{p^{N}-1} \cdots \sum_{x_{k}=0}^{p^{N}-1}(-1)^{x_{1}+\cdots+x_{k}}\left[x_{1}+\cdots+x_{k}\right]_{q}^{m} q^{-x_{1} m-\cdots-x_{k}(m+k-1)} \\
& =\frac{[2]_{q}^{k}}{(1-q)^{m}} \sum_{i=0}^{m}\left(\begin{array}{c}
m \\
i
\end{array}\right)(-1)^{i} \frac{1}{\left(1+q^{i-m}\right) \cdots\left(1+q^{i-m-k+1}\right)}
\end{aligned}
$$

where $\left(\begin{array}{c}m \\ i\end{array}\right)=m \cdots(m-k+1) / k !$

Note that $\lim _{q \rightarrow 1} G_{m, q}^{(-m, k)}=G_{m}^{(k)}$, where $G_{m}^{(k)}$ are the ordinary Genocchi numbers of order $k$ defined as

$$
\left(\frac{2 t}{e^{t}+1}\right)^{k}=\sum_{n=0}^{\infty} G_{n}^{(k)} \frac{t^{n}}{n !}
$$

By (2.4) and (2.5), we can obtain the following theorem.

Theorem 2.3. Let $m \in \mathbb{N}$. Then one has

$$
\frac{G_{m+1, q}^{(-m, 1)}}{m+1}=\sum_{i=0}^{m}\left(\begin{array}{c}
m \\
i
\end{array}\right) q^{x_{i}} \frac{G_{i+1, q}^{(-m, 1)}}{i+1}[x]_{q}^{m-i}=\frac{[2]_{q}}{(1-q)^{m}} \sum_{j=0}^{m} q^{i x}\left(\begin{array}{c}
m \\
i
\end{array}\right) \frac{(-1)^{j}}{\left(1+q^{j-m}\right)} .
$$

It is easily to check that

$$
\begin{aligned}
\frac{G_{n+1, q}^{(-n, 1)}}{n+1} & =\int_{\mathbb{Z}_{p}} q^{-(n+1) t}[x+t]_{q}^{n} d \mu_{q}(t) \\
& =\frac{1+q}{1+q^{d}}[d]_{q}^{n} \sum_{x=0}^{d-1}(-1)^{i} q^{-n i} \int_{\mathbb{Z}_{p}} q^{-(n+1) d t}\left[\frac{x+i}{d}+t\right]_{q^{d}}^{n} d \mu_{q^{d}}(t),
\end{aligned}
$$

where $n, d \in \mathbb{N}$ with $d \equiv 1(\bmod 2)$. Thus we have the following theorem.

Theorem 2.4. Let $d, n \in \mathbb{N}$ with $d \equiv 1(\bmod 2)$. Then for all $x \in \mathbb{Z}_{p}$,

$$
\frac{G_{n+1, q}^{(-n, 1)}(x)}{n+1}=\frac{1+q}{1+q^{d}}[d]_{q}^{n} \sum_{i=0}^{n}(-1)^{i} q^{-n i} G_{n+1, q}^{-n, 1}\left(\frac{x+i}{d}\right) .
$$

We note that if we take $x=0$, then we have

$$
\frac{G_{m+1, q}^{(-m, 1)}}{m+1}=\frac{1+q}{1+q^{m}} \sum_{k=0}^{m}\left(\begin{array}{c}
m \\
k
\end{array}\right)[n]_{q}^{k} \frac{G_{k+1, q^{n}}^{(-m, 1)}}{k+1} \sum_{j=0}^{n-1}(-1)^{j} q^{-(m-k) j}[j]_{q}^{m-k}
$$


where $n=1(\bmod 2)$. By $(2.10)$, we easily see that

$$
\frac{G_{m+1, q}^{(-m, 1)}}{m+1}-\frac{1+q}{1+q^{n}}[n]_{q}^{m} \frac{G_{m+1, q}^{(-m, 1)}}{m+1}=\frac{1+q}{1+q^{m}} \sum_{k=0}^{m-1}[n]_{q}^{k} \frac{G_{k+1, q^{n}}^{(-m, 1)}}{k+1} \sum_{j=0}^{n-1}(-1)^{j} q^{-(m-k) j}[j]_{q}^{m-k}
$$

Note that $\lim _{q \rightarrow 1} G_{m, q}^{(-m, 1)}=G_{m}$, where $G_{m}$ are the $m$ th Genocchi numbers defined as

$$
\frac{2 t}{e^{t}+1}=\sum_{n=0}^{\infty} G_{n} \frac{t^{n}}{n !}
$$

From (2.11), we can see that

$$
\left(1-n^{m}\right) \frac{G_{m+1}}{m+1}=\sum_{k=0}^{m-1}\left(\begin{array}{l}
m \\
k
\end{array}\right) n^{k} \frac{G_{k+1}}{k+1} \sum_{j=0}^{n-1}(-1)^{j} j^{m-k} .
$$

Let $F_{q}(t, x)$ be the generating function of $G_{m, q}^{(-m, 1)}$ as follows:

$$
F_{q}(t, x)=\sum_{n=0}^{\infty} \frac{G_{n+1, q}^{(-n, 1)}(x)}{n+1} \frac{t^{n}}{n !}
$$

By (2.7) and (2.14), we see that

$$
\begin{aligned}
F_{q}(t, x) & =\sum_{k=0}^{\infty}\left((1+q) \sum_{n=0}^{\infty}(-1)^{n} q^{-k n}[n+x]_{q}^{k}\right) \frac{t^{k}}{k !} \\
& =(1+q) \sum_{n=0}^{\infty}(-1)^{n} \sum_{n=0}^{\infty}(-1)^{n} \sum_{k=0}^{\infty} q^{-k n}[n+x]_{q}^{k} \frac{t^{k}}{k !} \\
& =(1+q) \sum_{n=0}^{\infty}(-1)^{n} e^{[n+x]_{q} q^{-n} t} .
\end{aligned}
$$

By (2.14) and (2.15), we can obtain the following theorem.

Theorem 2.5. Let $m \in \mathbb{N}$. Then for all $x \in \mathbb{Z}_{p}$,

$$
\frac{G_{m+1, q}^{(-m, 1)}(x)}{m+1}=[2]_{q} \sum_{n=0}^{\infty} q^{-n m}(-1)^{n}[n+x]_{q}^{m}
$$

\section{Acknowledgment}

This paper was supported by KOSEF (2009-0073396, 2009-A419-0065). 


\section{References}

[1] T. Kim, "On the multiple $q$-Genocchi and Euler numbers," Russian Journal of Mathematical Physics, vol. 15, no. 4, pp. 481-486, 2008.

[2] T. Kim, "Note on the Euler q-zeta functions," Journal of Number Theory, vol. 129, no. 7, pp. 1798-1804, 2009.

[3] T. Kim, "q-Bernoulli numbers and polynomials associated with Gaussian binomial coefficients," Russian Journal of Mathematical Physics, vol. 15, no. 1, pp. 51-57, 2008.

[4] T. Kim, "Some identities on the $q$-Euler polynomials of higher order and $q$-Stirling numbers by the fermionic $p$-adic integral on $\mathbb{Z}_{p}$, " Russian Journal of Mathematical Physics, vol. 16, pp. 501-508, 2009.

[5] T. Kim, "Note on Dedekind type DC sums," Advanced Studies in Contemporary Mathematics, vol. 18, no. 2, pp. 249-260, 2009.

[6] T. Kim, "A note on some formulae for the q-Euler numbers and polynomials," Proceedings of the Jangjeon Mathematical Society, vol. 9, no. 2, pp. 227-232, 2006.

[7] T. Kim, "A note on the generalized q-Euler numbers," Proceedings of the Jangjeon Mathematical Society, vol. 12, no. 1, pp. 45-50, 2009.

[8] T. Kim, "Note on the $q$-Euler numbers of higher order," Advanced Studies in Contemporary Mathematics, vol. 19, no. 1, pp. 25-29, 2009.

[9] Y.-H. Kim, W. Kim, and C. S. Ryoo, "On the twisted $q$-Euler zeta function associated with twisted q-Euler numbers," Proceedings of the Jangjeon Mathematical Society, vol. 12, no. 1, pp. 93-100, 2009.

[10] H. Ozden, Y. Simsek, S.-H. Rim, and I. N. Cangul, "A note on p-adic $q$-Euler measure," Advanced Studies in Contemporary Mathematics, vol. 14, pp. 233-239, 2007.

[11] Y. Simsek, "Generating functions of the twisted Bernoulli numbers and polynomials associated with their interpolation functions," Advanced Studies in Contemporary Mathematics, vol. 16, no. 2, pp. 251$278,2008$.

[12] Y. Simsek, V. Kurt, and D. Kim, "New approach to the complete sum of products of the twisted $(h, q)$-Bernoulli numbers and polynomials," Journal of Nonlinear Mathematical Physics, vol. 14, no. 1, pp. 44-56, 2007.

[13] M. Cenkci, Y. Simsek, and V. Kurt, "Further remarks on multiple $p$-adic $q$-L-function of two variables," Advanced Studies in Contemporary Mathematics, vol. 14, no. 1, pp. 49-68, 2007.

[14] T. Kim, "q-Volkenborn integration," Russian Journal of Mathematical Physics, vol. 9, no. 3, pp. 288-299, 2002.

[15] T. Kim, "On Euler-Barnes multiple zeta functions," Russian Journal of Mathematical Physics, vol. 10, no. 3, pp. 261-267, 2003.

[16] T. Kim, "Analytic continuation of multiple q-zeta functions and their values at negative integers," Russian Journal of Mathematical Physics, vol. 11, no. 1, pp. 71-76, 2004.

[17] T. Kim, "Power series and asymptotic series associated with the $q$-analog of the two-variable $p$-adic L-function," Russian Journal of Mathematical Physics, vol. 12, no. 2, pp. 186-196, 2005.

[18] T. Kim, "Multiple p-adic L-function," Russian Journal of Mathematical Physics, vol. 13, no. 2, pp. 151$157,2006$.

[19] T. Kim, "A note on $p$-adic $q$-integral on $Z_{p}$ associated with $q$-Euler numbers," Advanced Studies in Contemporary Mathematics, vol. 15, pp. 133-138, 2007.

[20] T. Kim, "On $p$-adic interpolating function for $q$-Euler numbers and its derivatives," Journal of Mathematical Analysis and Applications, vol. 339, no. 1, pp. 598-608, 2008.

[21] T. Kim, "On the analogs of Euler numbers and polynomials associated with $p$-adic $q$-integral on $\mathbb{Z}_{p}$ at $q=-1, "$ Journal of Mathematical Analysis and Applications, vol. 331, no. 2, pp. 779-792, 2007.

[22] T. Kim, "A note on $p$-adic $q$-integral on $\mathbb{Z}_{p}$ associated with $q$-Euler numbers," Advanced Studies in Contemporary Mathematics, vol. 15, no. 2, pp. 133-137, 2007.

[23] T. Kim, " $q$-Euler numbers and polynomials associated with $p$-adic $q$-integrals," Journal of Nonlinear Mathematical Physics, vol. 14, no. 1, pp. 15-27, 2007.

[24] A. Yu. Khrennikov, p-adic Valued Distributions and Their Applications to the Mathematical Physics, Kluwer Academic Publishers, Dordrecht, The Netherlands, 1994.

[25] A. Yu. Khrennikov, Non-Archimedean Analysis: Quantum Paradoxes, Dynamical Systems and Biological Models, vol. 427 of Mathematics and Its Applications, Kluwer Academic Publishers, Dordrecht, The Netherlands, 1997.

[26] A. Yu. Khrennikov, Interpretations of Probability, VSP, Utrecht, The Netherlands, 1999. 